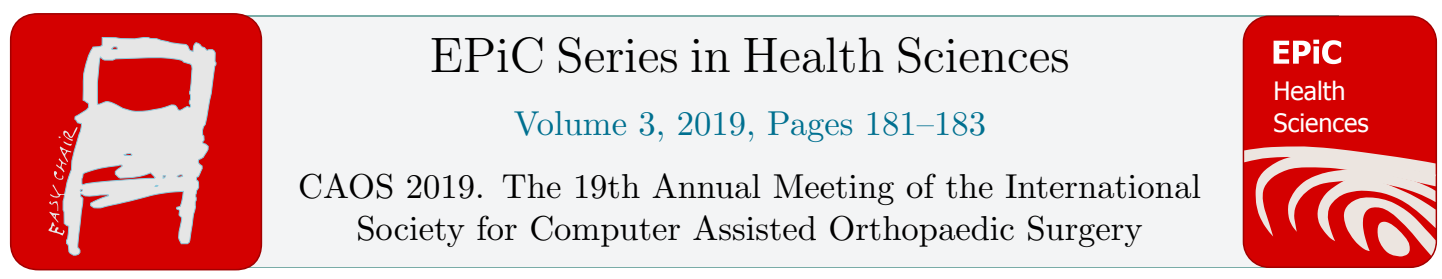

\title{
Handle Navigation for a Smart Handheld Robot
}

\author{
Shuo-Suei Hung ${ }^{1}$ and Ping-Lang Yen ${ }^{2 *}$ \\ ${ }^{1}$ Taipei Tzu Chi Hospital, New Taipei City, Taiwan \\ ${ }^{2}$ National Taiwan University, Taipei, Taiwan \\ Hung_ortho@yahoo.com.tw, plyen@ntu.edu.tw
}

\begin{abstract}
We demonstrated a miniature handheld robot in assisting the surgeon to create a tunnel during pedicle screw fixation. The robot is aimed at stabilizing the drill during penetrating the surface of a vertebra and reducing the drill drifting from the entry point caused by involuntary tremor. The handheld robot is consisted of a hexapod platform. The master control was designed to cooperate with the 6-DOF parallel slave robot. The human machine interface has been investigated for the drill to target the planned trajectory with the operator's handle motion.
\end{abstract}

\section{Introduction}

Medical robots served to assist surgical intervention have been widely adopted in many indications of use. Two main categories of general and special purpose robotic systems are presented. The general purpose robotic system offers versatile functionalities, such as suction, cutting and sewing etc through its accurate and dexterous motion and high quality of anatomical images [1]. These attractive functions, however, are hampered by the large footprint of the robot so that the general robotic system cannot be install inside a crowded operation room, thus usually require a dedicated operation room for the whole system. The installation leads to be very expensive and confines the usage within institutes which can afford the expenses. On the other hand, the special purpose robotic system only provide specific functions, such as a guide for conventional tools, or drilling or cutting along the surgical planning path etc. Among these are the bone mounted robot [2], or handheld robot [3], which have the superiority of compact installation space and compatible to daily surgical procedure. A miniature handheld robot is proposed to assist the surgeon to create a tunnel during pedicle screw fixation. However the coordination between the operator and the handheld robot may become an issue if the human machine interface is not appropriated designed. In this paper the human machine interface associated with the master hand control will be presented.

\footnotetext{
${ }^{*}$ Corresponding Author
} 


\section{Method}

The overall controller for the handheld robot constitutes two sub-modules of the master and the slave respectively. The graphic display module is for the master and the robot control module is for the slave. The graphic display module is aimed at guiding the master handle hold by the operator (1) to approach the targeted anatomy, (2) to confine the base of the slave robot within the range so that the target path of the tool is within the workspace of the slave robot. Two proposed graphic displays were compared for the navigation purposes in terms of targeting the pre-defined path. Figure 1(a) shows the traditional navigation arrangement. The drilling tool (a cross in blue color) is displayed relative to the target path (a stick in black color). The positioning errors of the drilling tool relative to the target path were used to adjust the handle gradually and finally aiming at matching the target. Figure 1(b) shows the master handle relative to the target path. This allows the surgeon to recognise how to move the master handle toward to the target path.
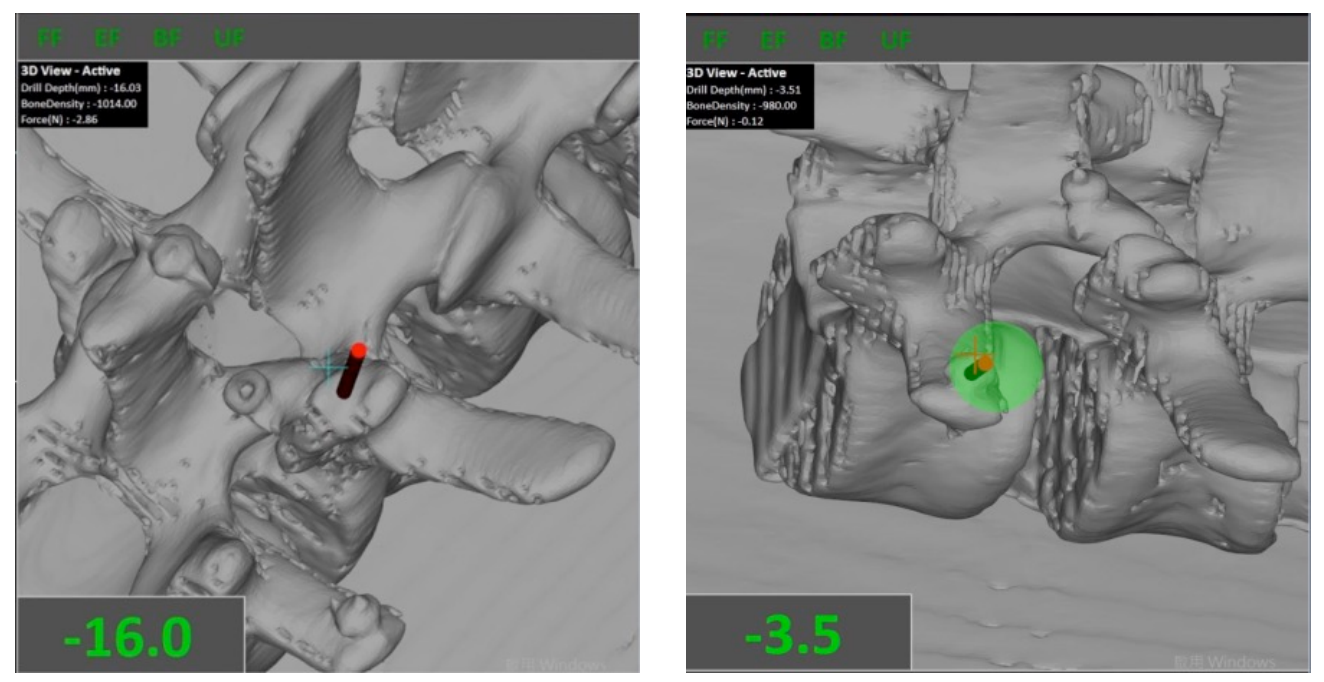

Figure 1: Human machine interface design (a) Tool (a blue cross) relative to the target path (a black stick) (b) handle (a green circle) to the target path

\section{Result and Discussion}

The handheld robot system was tested to investigate the effects of two graphic displays on the drilling stabilization to target entry point of an anatomy. The T5 (\#5 Thoracic) from a human CT model was chosen and its 3D printing model was generated. Experiments were performed to verify the capability of stabilizing the drill bit to the target trajectory. Figure 2 shows that the positioning errors was larger under tool-based navigation graphics than handle-based navigation. The hand motion also wandered in a larger range in tool-based graphics. The handle-based graphics could assist the operator to control the handle in cooperating with the robot along the target path. 

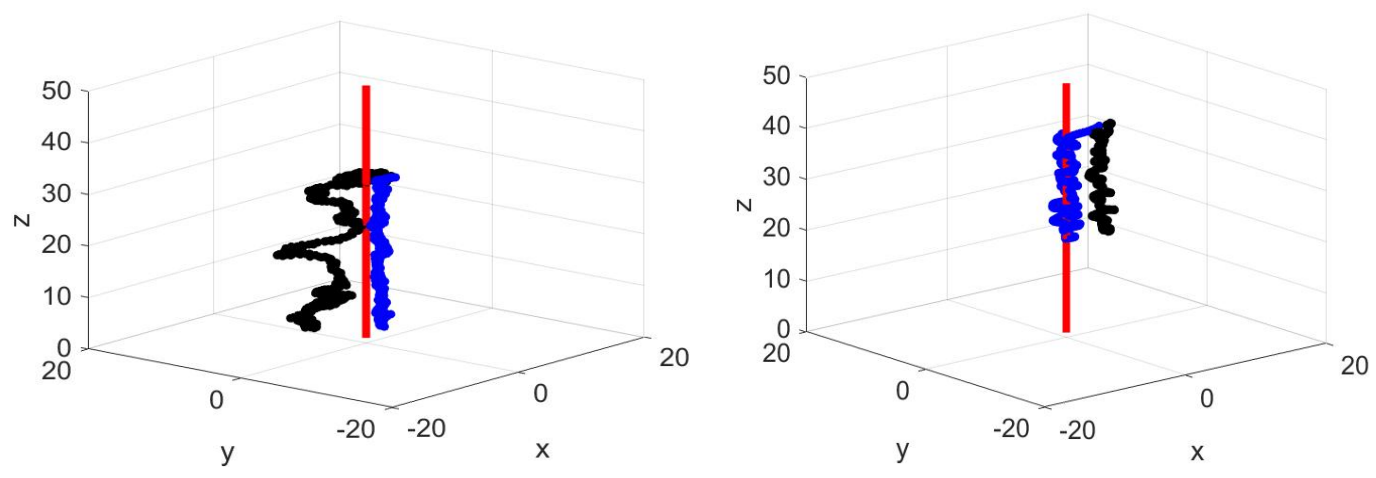

Figure 2: The movement path of master hand (black line) and drilling tool (blue line) relative the target path (red line) (a) Tool-based navigation graphics (b) Handle-based navigation graphics

\section{Conclusion}

The handheld robot is under the telemanipulator architecture of master on the slave and implements collaborative integration between the human operator and the robot. The experimental results shows that proper design of human machine interface for the master handle to co-work with the robot is crucial for the drill to target the desired path.

\section{References}

[1] Davies, B. review of robotics in surgery, Proceedings of the Institution of Mechanical Engineers, Part H: Journal of Engineering in Medicine 214(1):129-140, 2000.

[2] Shoham, M., M. Burman, E. Zehavi, L. Joskowicz, E. Batkilin, and Y. Kunicher, Bonemounted miniature robot for surgical procedures: Concept and clinical applications. IEEE Transactions on Robotics and Automation 19(5):893-901, 2003.

[3] Ang, W. T., P. Pradeep, and C. Riviere. Active tremor compensation in microsurgery, IEEE International Conference of 26th Annual Engineering in Medicine and Biology Society, 2004. 